\title{
A Classical LTE Cellular System Simulator for Computer Network Education
}

\author{
Julius Yaw Ludu $(\mathbb{D}$, Justice Kwame Appati $(\mathbb{D}$, Ebenezer Owusu $(\mathbb{D}$, \\ and Prince Boakye-Sekyerehene
}

Department of Computer Science, University of Ghana, Legon, Accra, Ghana

Correspondence should be addressed to Justice Kwame Appati; jkappati@ug.edu.gh

Received 14 September 2021; Accepted 2 November 2021; Published 15 November 2021

Academic Editor: Noé López Perrusquia

Copyright (c) 2021 Julius Yaw Ludu et al. This is an open access article distributed under the Creative Commons Attribution License, which permits unrestricted use, distribution, and reproduction in any medium, provided the original work is properly cited.

\begin{abstract}
The proposal of LTE in the standardization of cellular network systems has received considerable attention in the research domain, and most subscribers widely use it. Despite the enormous acceptance of the system, academia as an industry is usually disadvantaged in training students due to the cost implication in setting up a prototype. In bridging this gap, simulators are traditionally developed as a testbed to aid students appreciate how these systems work. Although there are several simulators available on the market, these simulators are quite expensive to acquire while others come with license restrictions. In this study, a classical LTE cellular system simulator is proposed as a testbed to aid the education of computer networks at college. The proposed simulator is an extension of the functionality of LTE-Sim frameworks. Usability testing of the proposed study reveals that the system is much easier to simulate the various scenarios in wireless communication.
\end{abstract}

\section{Introduction}

Education is essential and serves as the foundation for the growth of every human being. Its effects are obvious not only in childhood, but also in maturity. Every educational institution's priority is hinged on providing appropriate motivation and high-quality standards. Educational resources are one of the methods that may be utilized to supplement the educational process. Students can be motivated to learn if they have access to appropriate materials [1]. As computer science and technology advances, several computing courses and curriculums have been developed and made available on the internet. The majority of them are very self-sufficient, which creates issues such as a new learner's inability to quickly access suitable curriculums and courses without further help [2]. Furthermore, many colleges do not have the funds to create state-of-the-art labs and keep same up to date on a regular basis. Owing to the limits of real-world equipments, it has become difficult to carry out all types of experimentation in the real lab. To tackle these issues, several colleges now use computer network simulator (CNS) software. This helps simulate numerous types of network equipment and carries out a wider range of experiments. Students leverage on these CNS to conduct their experiment, much like they would be in a real lab [3].

In a few decades, the world at large has experienced a steeper increase in how data is used in a cellular ecosystem [4-6]. As the drive for a more enhanced technology evolves in this space, the demand for a higher data rate, high connectivity speed, power efficiency, and low cost in application use also increases [7]. More cellular technologies are being developed in response to the varying need of users in the telecommunication industry. Among these technologies, the long-term evolution (LTE) developed by the $3^{\text {rd }}$ Generational Partnership Project (3GPP) is proposed to cater for the evergrowing demand in the use of data $[8,9]$. Historically, digital communication was birth in the ' 90 s as an offshoot of the fixed wired telephony industry. This was in the form of 2 G GSM, which was primarily for voice service with data service ranked low on the priority queue [10]. 


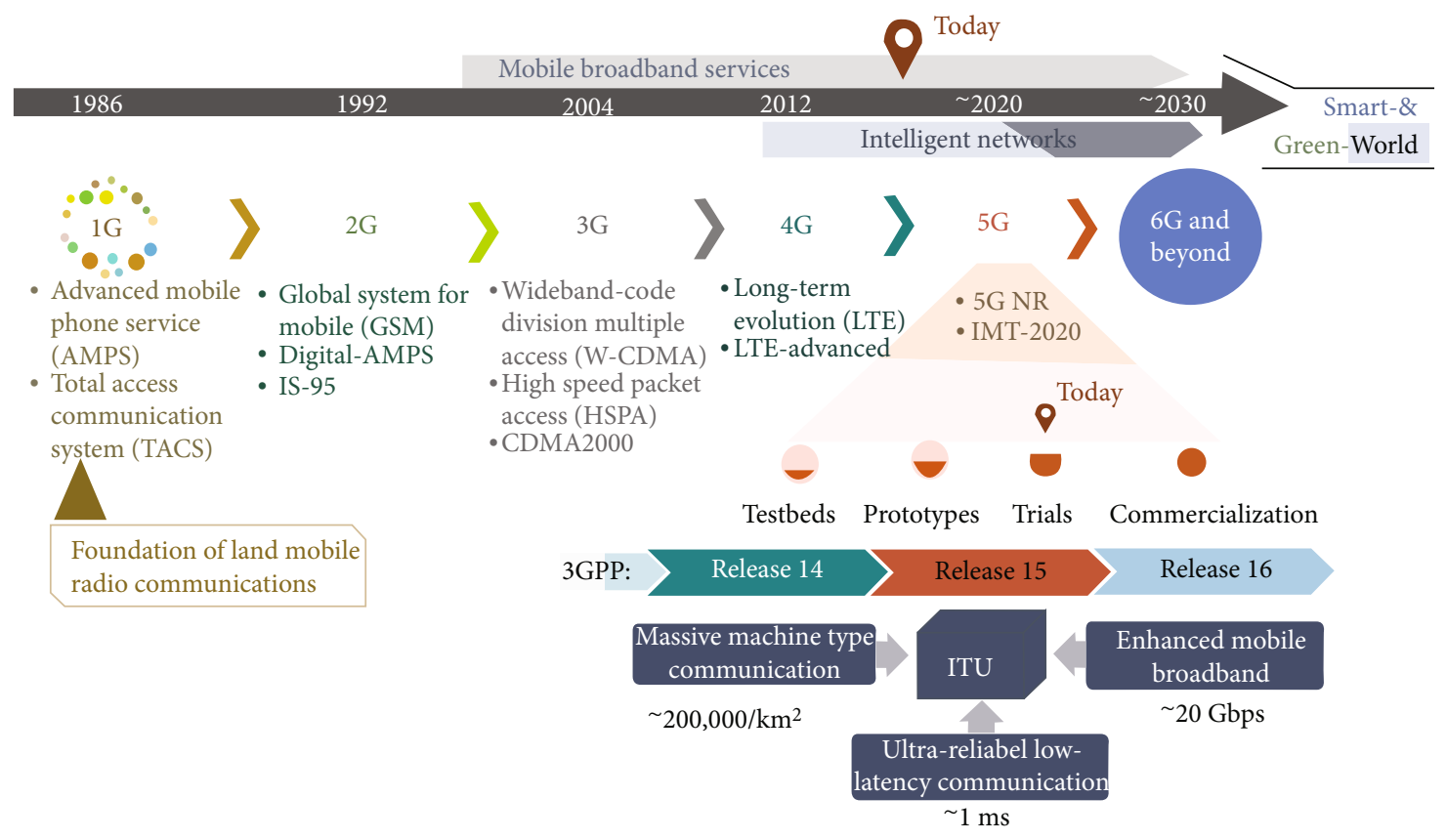

Figure 1: Trends in cellular network systems [13].

Other services like SMS for text messaging and packetized data through GPRS and HSPA were made possible in later years $[11,12]$. Figure 1 shows the trend of how cellular networks have fed over the years.

With the introduction of LTE, an entirely new air interface on an all-IP technology based on Orthogonal Frequency Division Multiplexing (OFDM) was implemented, setting the basis for a $4 \mathrm{G}$ capable mobile communication technology [14] which is known to be more spectrally efficient. Thus, it can deliver more bits per Hertz, providing an economy of scale and spectrum reuse [15]. Some other benefits derived from LTE is the smooth integration and handover to and from existing 3GPP networks. It also supports full mobility and global roaming and ensures that operators can deploy while bringing subscribers to a true mobile broadband ( $\sim-10 \mathrm{Mb} / \mathrm{s} / \sim 15 \mathrm{~ms}$ latency), enabling a quality video experience and media mobility [16]. With improved capacity, speed, and latency, LTE made accessing applications faster and enabled a wealth of new applications previously available only on a wired Internet connection. This has necessitated the need to appreciate this technology in both a wet and dry lab. Over the years, the simulation method has been adopted as the standard mechanism for analyzing and evaluating the performance of an emerging protocol or model [17]. For the purpose of usability and network education, these models and protocols are implemented through graphical user interfaces (GUI). Currently, there are very few simulation environments that have been developed for use in computer science (CS) education in the area or networks to mimic the performance of LTE systems [3, 18, 19]. Unfortunately, the few that exists comes with a commercial license, making it unsuitable for training in an academic environment. Other simulation environments are made publicly available through the open-source movement, but this also comes in a more sophisticated package with lit- tle to no user guide, which complicates its use as an introductory course at the tertiary level $[20,21]$.

In this study, we proposed an LTE simulator with the functional ability to perform standard network scenarios and further extend the capability of the LTE-Sim framework. To resolve the issue of usability, the proposed system is presented in the form of a GUI, which allows users to enter only the required parameters of a given network scenario selected. For ease of result interpretation in teaching and learning, the output of each scenario formulated is in the form of a graph. Comparatively, a quick survey demonstrates that the proposed system will serve the intended purpose in a classroom setup as opposed to other proposed systems. Subsequent sections of this study are structured as follow: Section 2 talks about the materials and methods used for this study. It gives an overview of the proposed platform and the system requirement. In the same section, we discuss the user interface requirement and the various parts of the system. In the third section, we have the system testing and discussion where a test scenario is demonstrated to verify the proposed system functions. Finally, we end with a conclusion.

\section{Materials and Methods}

2.1. System Overview. In this study, five (5) major components are required to build the proposed system. These components are the main simulation platform, the LTE-Sim file, graphs for simulation results, the parameter dialog, and tools containing some defined libraries. Figure 2 gives a schematic representation of the context diagram of the proposed system. From Figure 2, the main simulation platform is created when the system is executed. The main simulation platform houses the various simulation scenarios and most of the standard LTE dedicated simulations. Among the standard 


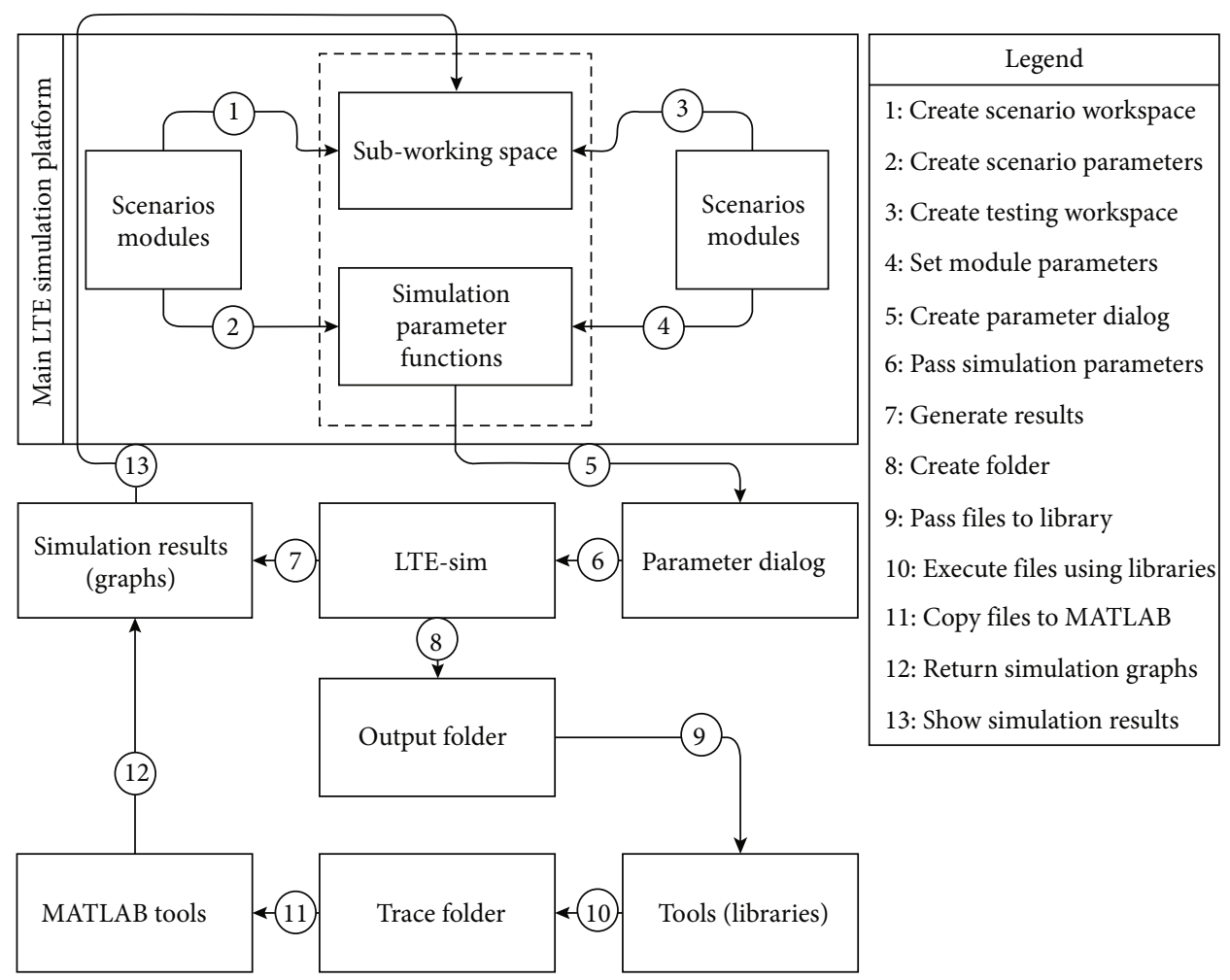

Figure 2: Context diagram of the proposed system.

scenarios are single cell, single cell with interference, single cell with streets, a single cell with Femto, and multicell. The system also has the ability to test for AMC mapping, SINR urban, mobility models, throughput urban, scalability test macro with Femto, throughput with building, throughput macro with Femto, and multicell SINR plot.

Executing a scenario or a dedicated test in the main simulation platform first creates a subworkspace followed by parameter initialization relating to the specific scenario to be performed. These parameters are editable using the parameter dialog with their outputs sent to the LTE-Sim module. The LTE-Sim module performs computation using the predefined functions and returns the required output as graphs for further analysis and interpretation. On the other hand, dedicated simulation is executed through the LTESim module resulting in files stored in an output folder. Appropriate libraries use up these files to produce MATLAB codes stored in the trace folder. These codes are executed to generate graphs that are displayed to users through the subworkspace.

2.2. System Specification and Functional Requirement. In this study, the Iterative Model Design (IMD) was leveraged to build the proposed architecture. This design paradigm was found appropriate for this study as it starts with a simple implementation of small components and iteratively improves the evolving versions until the system is complete and ready for deployment. At each iteration, there is an extra degree of freedom that permits the modification of the design and the creation of new functional capabilities [22].
From Section 2.1, each component is considered a build or block, and each is tested and implemented. The first build takes care of the main simulation area, and the second build considers the various parameter dialogs for various scenarios and testing modules. Build three is for the compilation of various functions used in the LTE-Sim.

Build four is the creation of tools as libraries to convert trace files into an appropriate structure for use in MATLAB. Finally, build five is for the display of outputs in the form of graphs. Figure 3 gives a schematic view of the IDM as applied in this study.

2.3. User Interface Requirement. This section explains, in brief, the interface requirement of the proposed system as follows:

(1) Users of the system should be presented with the main simulation page. The page should contain the main menu defined as File, Scenarios, Test, About, and Exit. The scenario menus should fire up the standard simulation scenarios for both single and multicells

(2) The test menu should present the dedicated test modules such as mobility model, test with Femto, scalability test, and test with buildings or streets

(3) The user should decide on either simulating a scenario or performing a test module

Figures 4 and 5 show the sample screens of the main simulation platform and the deducted test module. 


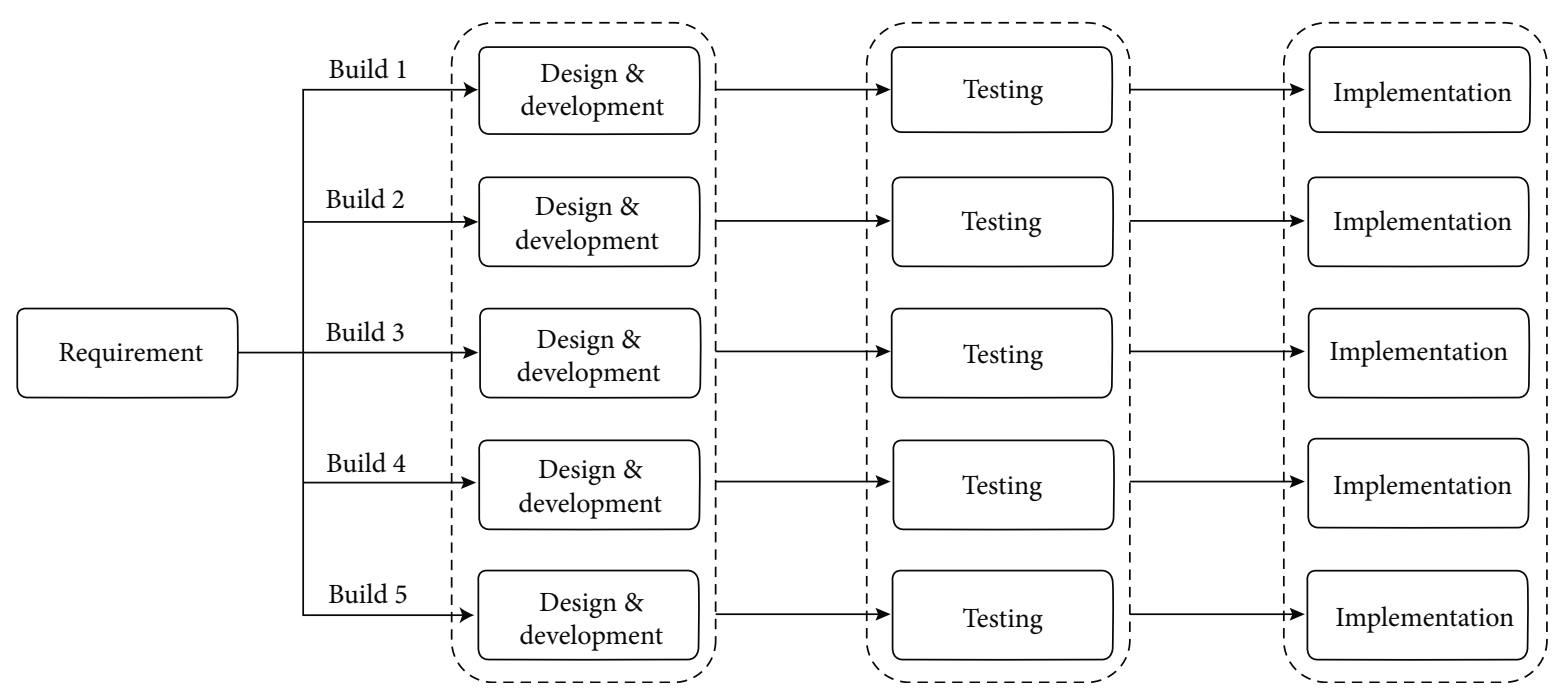

FIGURE 3: Iterative model design of the proposed system [23].

FIgURE 4: Main simulation platform.

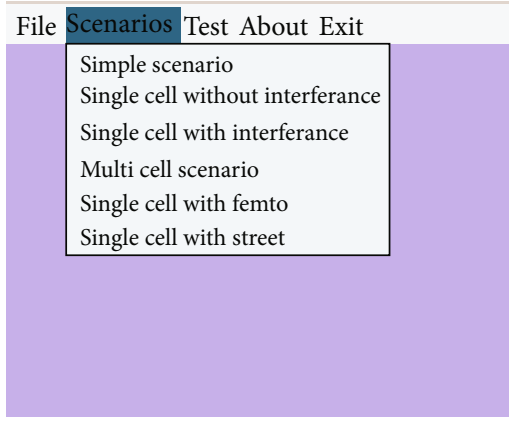

\begin{tabular}{|c|c|c|}
\hline \multirow{10}{*}{\multicolumn{2}{|c|}{ (c) File Scenarios }} & Test About Exit \\
\hline & & Test AMC mapping \\
\hline & & Test mobility model \\
\hline & & Scalability test macro with femto \\
\hline & & Test SINR (urban) \\
\hline & & Test throughput (urban) \\
\hline & & Test throughput macro with femto \\
\hline & & Test throughput building \\
\hline & & Test SINR (femto) \\
\hline & & Test Multicell SINR plot \\
\hline
\end{tabular}

Figure 5: A scenario menu and a testing menu. 


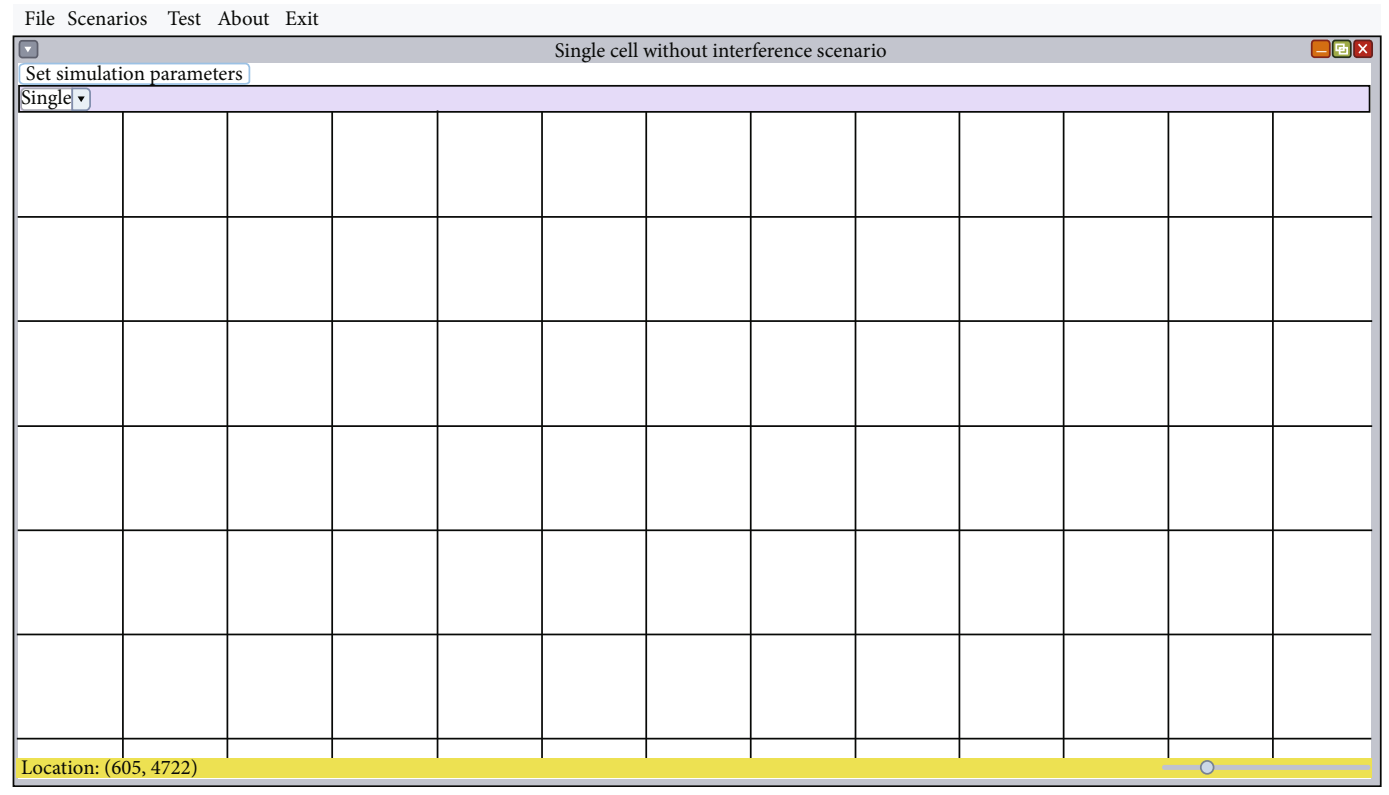

Figure 6: A workspace for single cell without interference.

2.4. Simulation Subworkspace. Figure 6 presents a view of the simulation subworkspace. In this workspace, the user is provided with an environment where they can either perform a scenario or run a test. A similar workspace is presented to users for all other scenarios or test modules depending on the activity selected. The workspace also interfaces with the parameter dialog to capture all required parameters for a given test or scenario.

2.5. Simulation Parameter Settings. This interface of the proposed system helps users define parameters for their test or scenario. The interface comes with two tabs, the initial parameter setup and other simulation parameters. The initial setup compartment presents default values to the user and allows them to alter them to their needs. Figure 7 is a demonstration of how the dialog functions.

The other parameter tab considers parameters such as the number of users' equipment, radius, video, number of VOIP, and best effort flows. In building this dialog, three schedulers were adopted, thus Modified Largest Weighted Delay First (M-LWDF), Exponential Proportional Fair (EXP), and Proportional Fair (PF). On executing these parameters, the user is present with seventeen (17) different graphs from four (4) different applications, thus VOIP, constant bit rate (CBR), video, and infinite buffer. These graphs also encompass some performance indexes such as system throughput, average delay, packet loss ratio (PLR), spectral efficiency, and fairness. Figure 8 gives a sample presentation of the spectral efficiency.

\section{System Testing and Discussion}

This section of the study explains the proposed system's validity and assesses the proposed system's potency and its usefulness to be used in college as an academic tool for computer networks. To start with, a single cell scenario without interference with a fixed eNodeB at the center of the cell is defined given that the users are moving at a speed of $3 \mathrm{kmph}$, and the mobility of each user equipment (UE) traveling cell is described with a random direction model. Assume that the users are moving in a bounded region equal to $1 \mathrm{~km}$, the number of connections to an eNodeB at a particular time varies from 10 to $100 \mathrm{UEs}$, and each UE receives one video flow, one VOIP flow, one constant bit rate, and one best effort flow at the same time. With the given assumptions, the performance result of the three different standard schemes is explored and evaluated in this section. The simulation of this scenario took $120 \mathrm{sec}$, and the comparison is divided into subgraphs based on the performance indices such as packet loss ratio (PLR). Figure 9 shows the throughput of the system over an increasing number of users. From the graph, it is observed that the system's throughput with respect to video trace degrades as the number of users increases. M-LWDF demonstrated a higher performance using the throughput metric over EXP/PF and PF schemes from the scenario.

Theoretically, MLWDF is known to prioritize real-time flows with the highest delay for their head of line packets and the best channel condition; hence, the better QoS requirements noted. It also supports multiple data users, which also results in a better performance in the throughput over EXP/PF and PF.

Figure 10 also shows the ratio of packet loss under the same schemes. From the graph, a significant difference is observed between these three schemes. The PF scheme recorded the highest PLR with increasing users, followed by $\mathrm{EXP} / \mathrm{PF}$ and M-LWDF. In video streaming applications, it is recommended that the packet loss ration threshold be kept below 0.01, which in effect makes M-LWDF more desirable.

In Figure 11, we have a plot of packet delay and the number of users to evaluate the performance of the three schemes. From the graph, we observed that the EXP/PF 


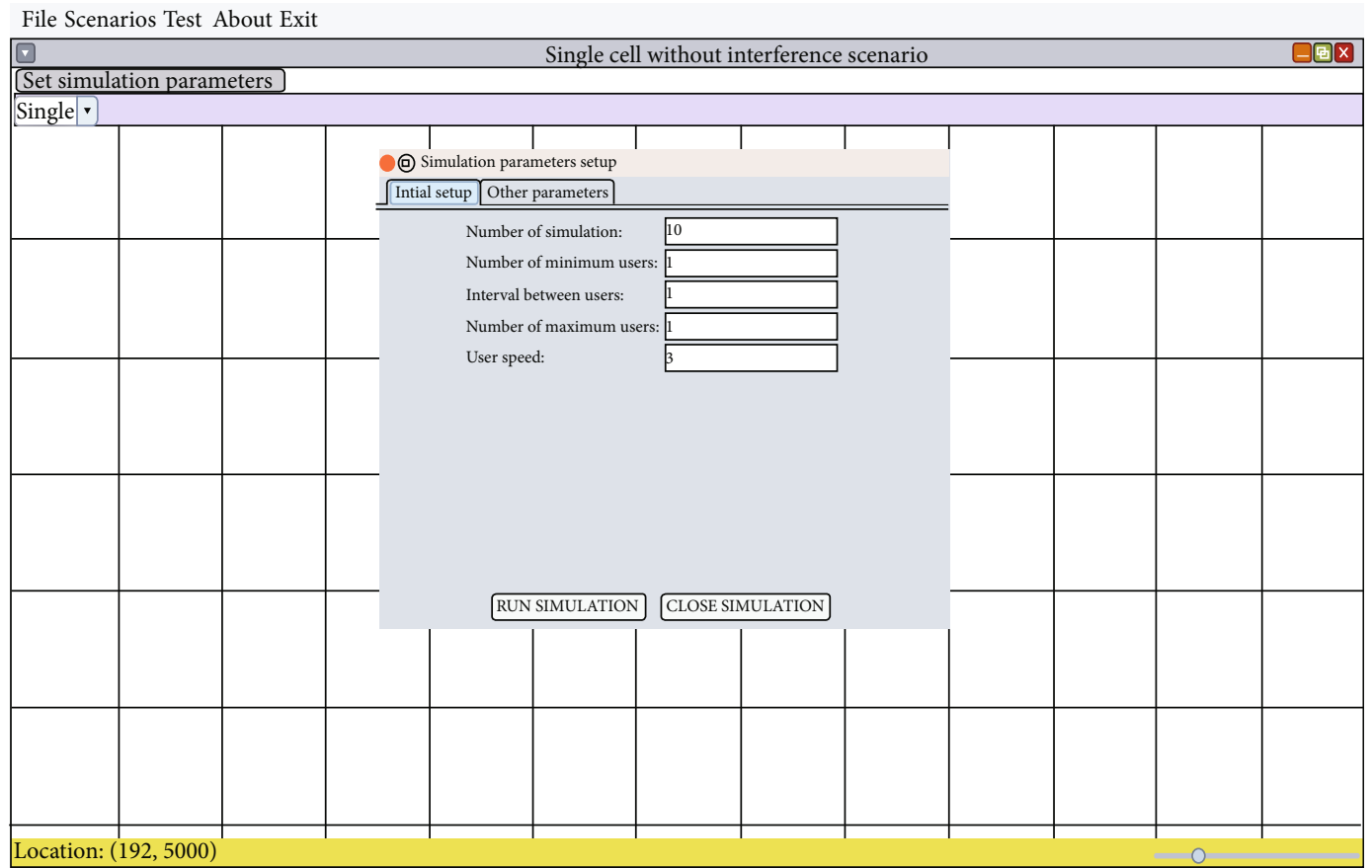

FIgURE 7: Initial simulation setup parameters.

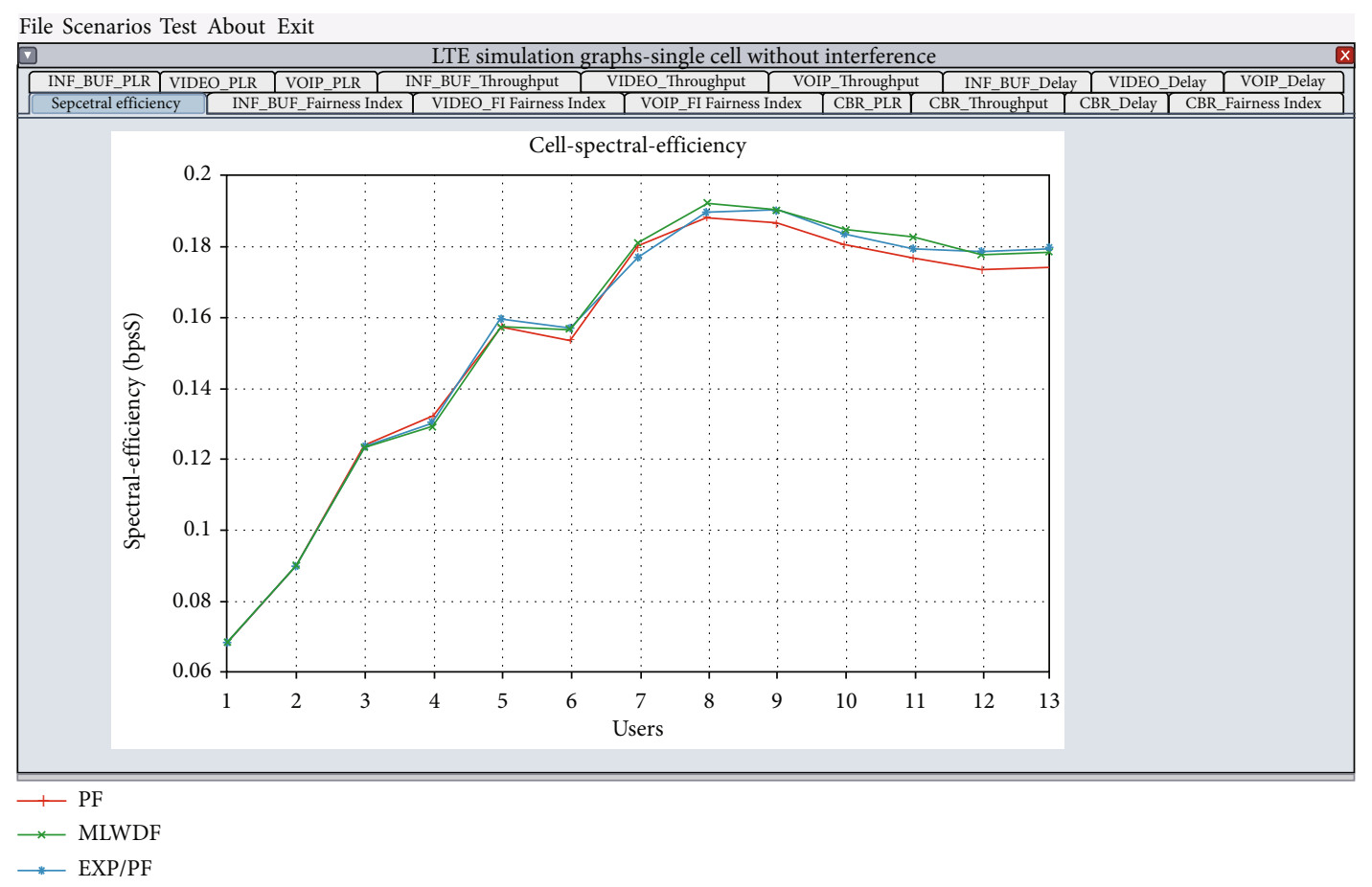

FIgURE 8: Spectral efficiency vs. number of users.

performs slightly better than M-LWDF and PF. This observation is usually attributed to the use of exponential function in the EXP/PF scheduler to delay targets in real-time traffics compared to the M-LWDF scheduler.

Observation in cell spectral efficiency is not different from that of the throughput since this was only run for video applications, as shown in Figure 12. The plot shows that the
M-LWDF scheme has a better spectral efficiency performance than EXP/PF and PF schemes.

Finally, we have Figure 13, which demonstrates the performance schedulers in assessing the fairness index of the scenario. From the graph, again, M-LWDF emerged better compared to EXP-PF and PF. Once again, this observation is attributed to the fact that the PF component responsible 


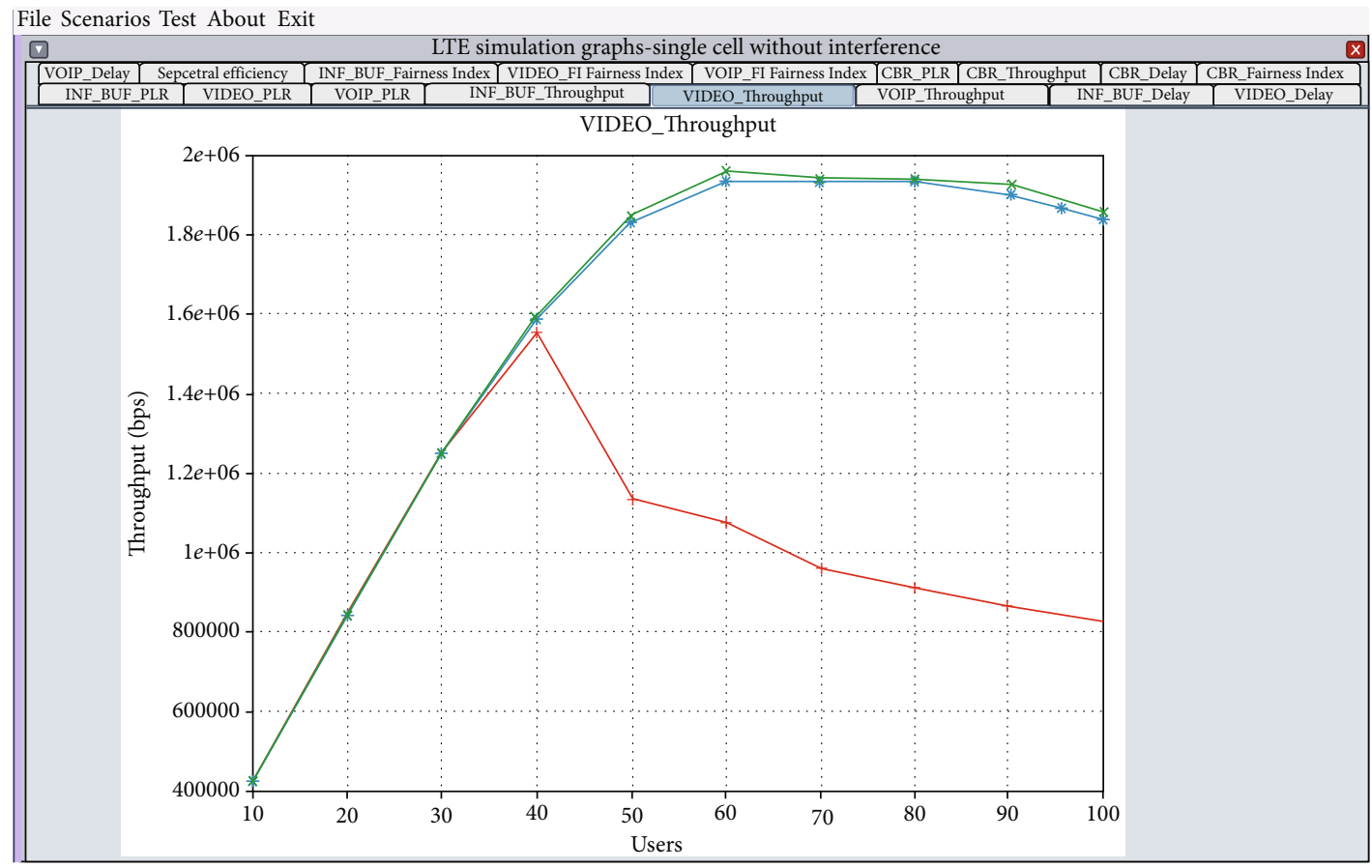

$+\mathrm{PF}$

$\rightarrow$ MLWDF

* $-\mathrm{EXP} / \mathrm{PF}$

Figure 9: System throughput vs. number of users.

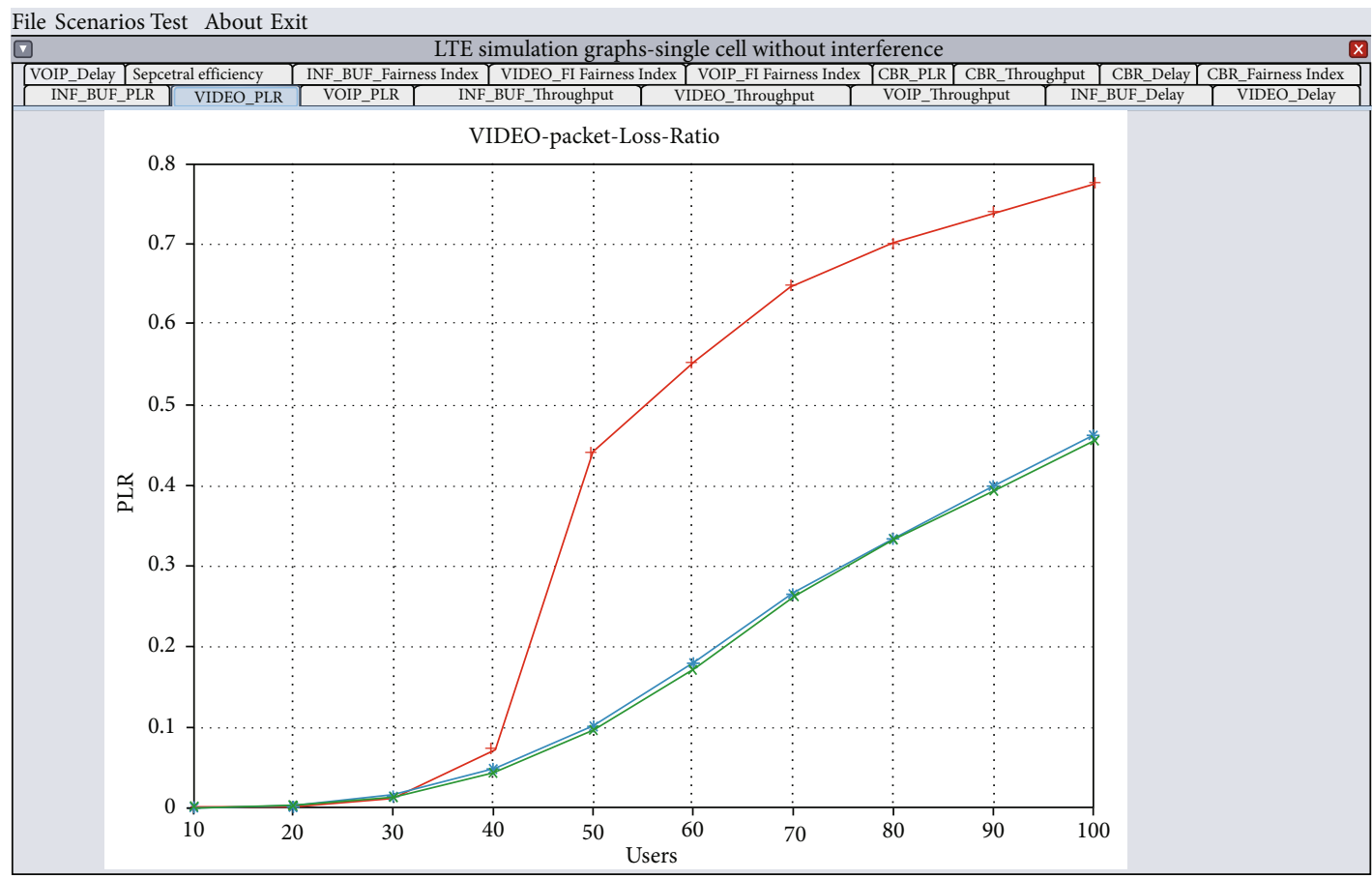

$+\mathrm{PF}$

$\star$ MLWDF

* EXP/PF

Figure 10: System packet loss vs. number of users. 


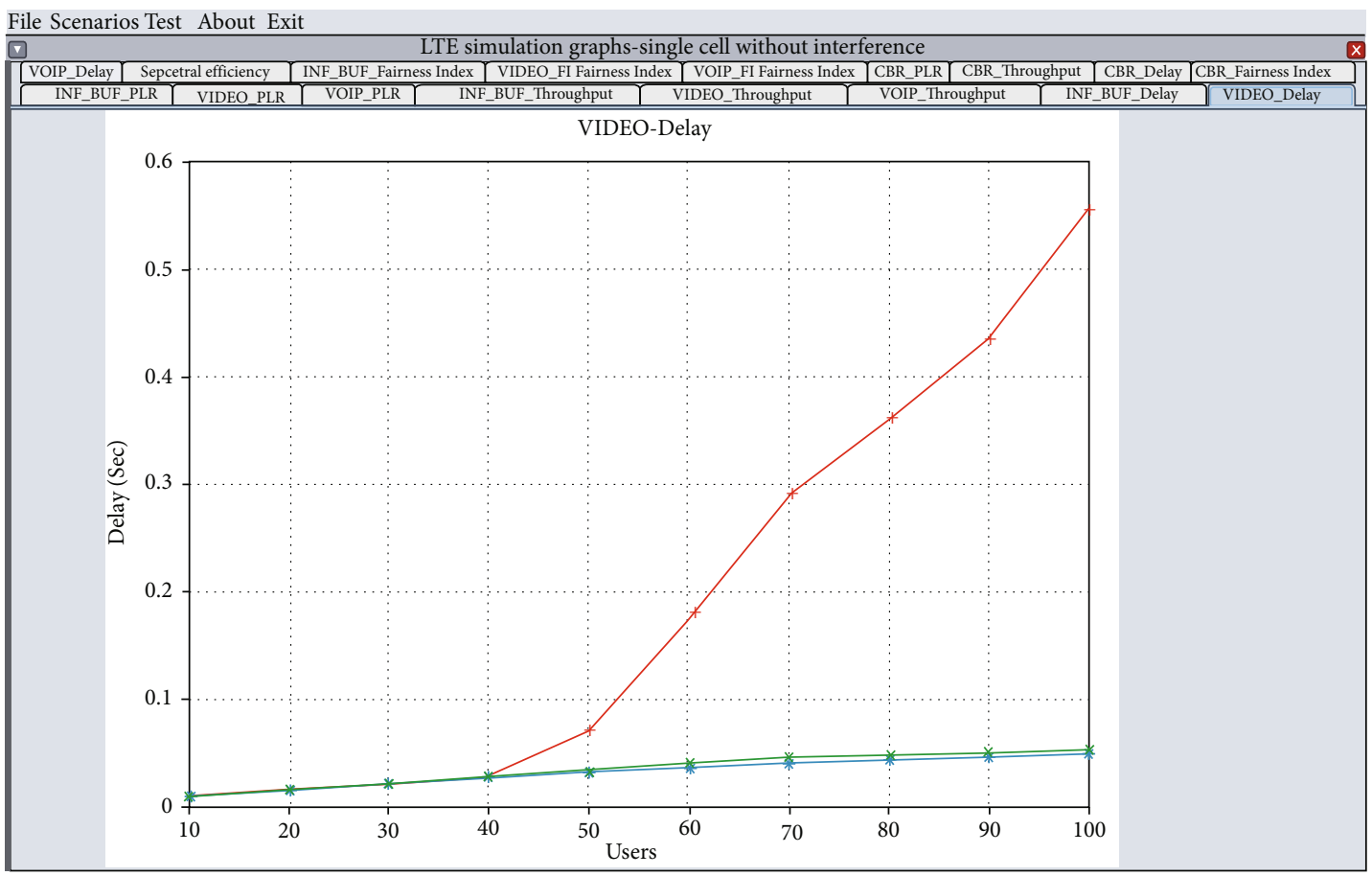

$+\mathrm{PF}$
$*$ MLWDF
$*$ EXP/PF

Figure 11: System packet delay vs. number of users.

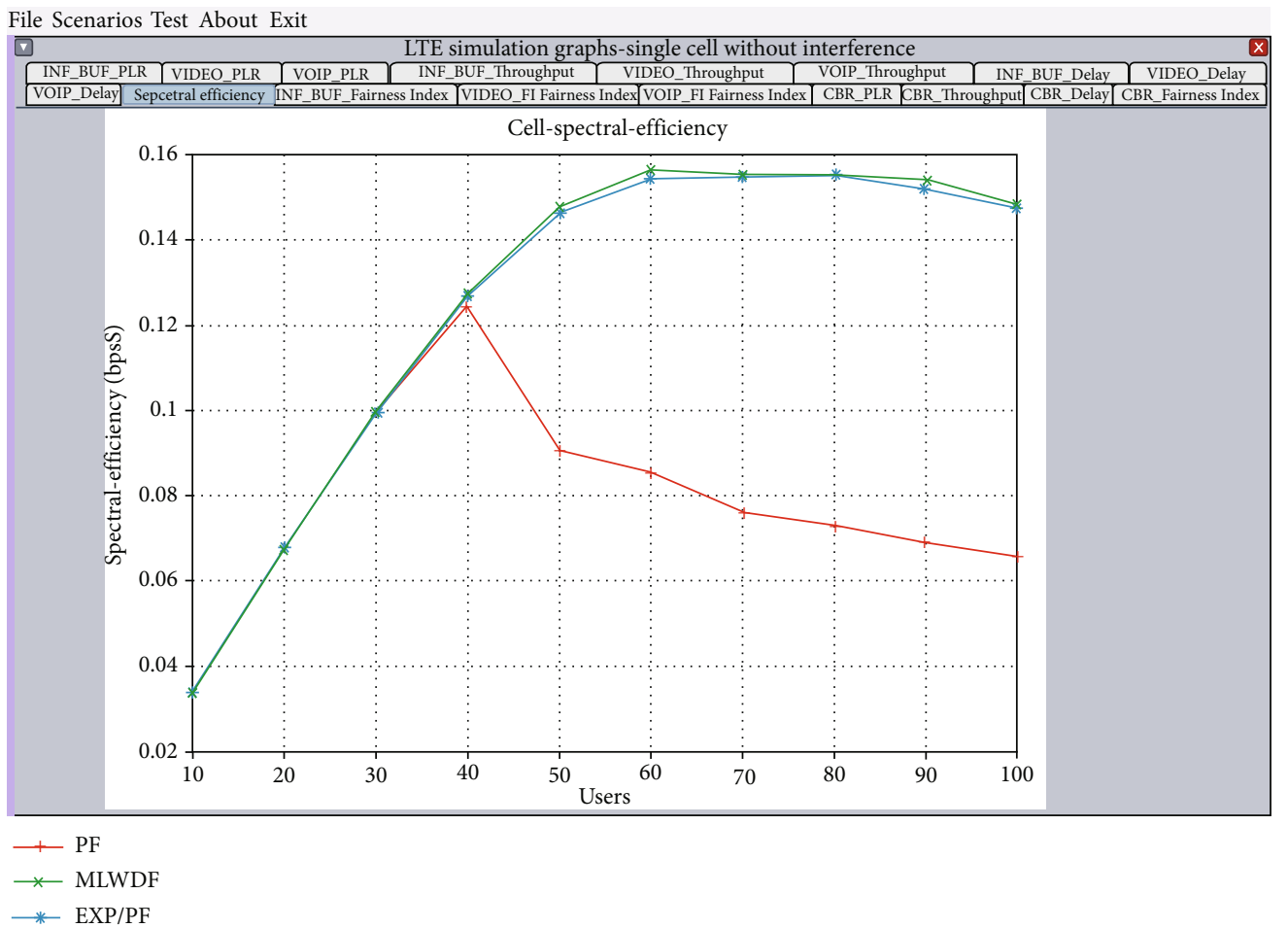

Figure 12: Spectral cell efficiency vs. number of users.

for fairness in the M-LWDF scheduler is more dominant compared to the QoS component. This unfortunately is not the case for EXP-PF since the QoS component only ensures more adherence to delay bound. In general, as the number of users increases, it is expected that the fairness index performance will also drop. 


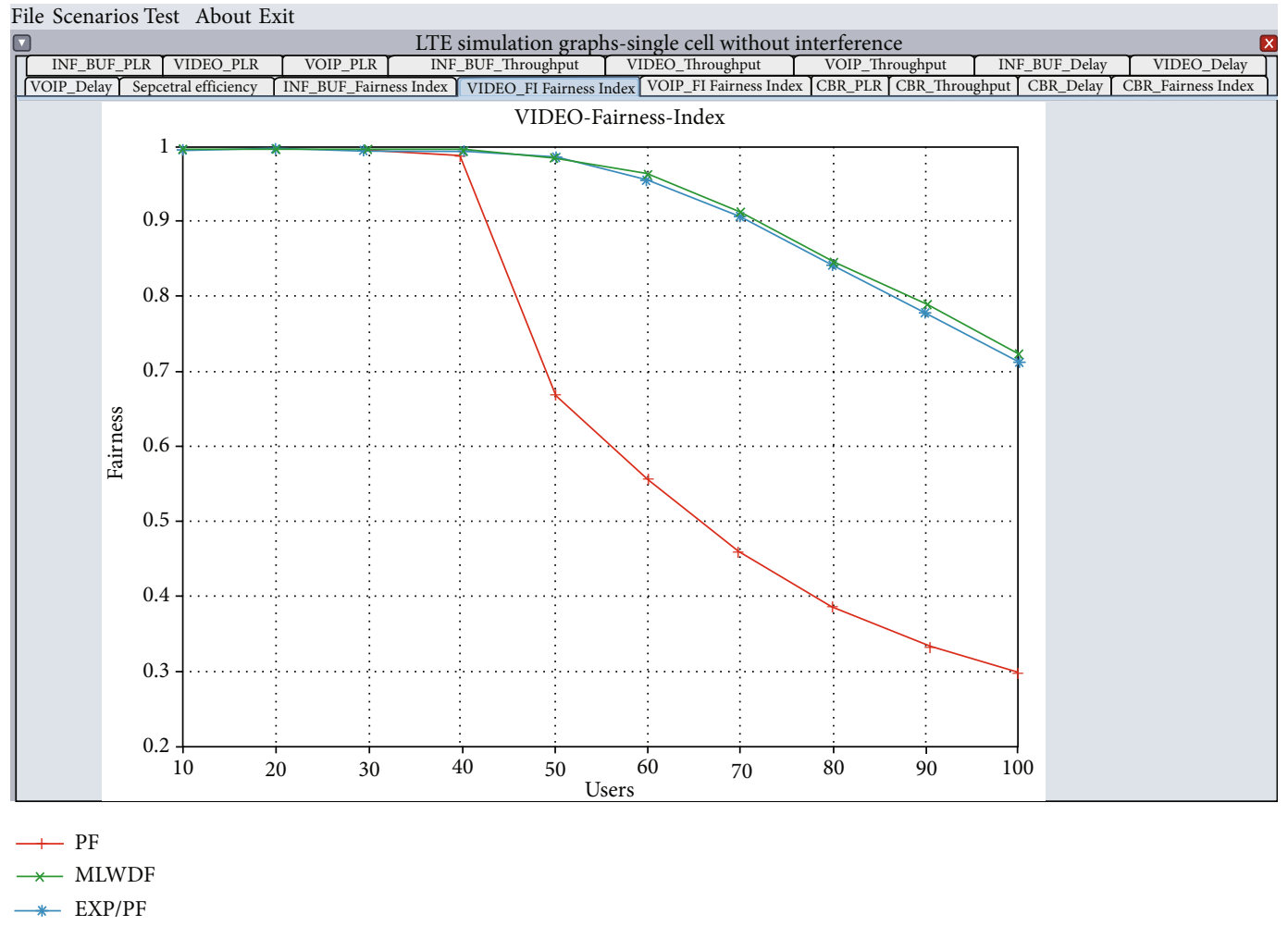

FIGURE 13: System fairness index vs. number of users.

\section{Conclusion}

In this study, we proposed a new testbed for the simulation of the LTE cellular system. This is to aid computer network education at the college level with a better appreciation of the subject matter. The proposed solution is able to simulate fairness index, the spectral cell efficiency, the packet delay, packet loss, and system throughput. It also provides a platform to manipulate parameter to fit a defined system requirement. The study further verifies the suitability and validity of the proposed framework in Section 3 using a sample off the shelf scenario. This serves as a foundational tool to practice all standard LTE modules. The study seeks to extend the proposed framework to support other more advanced cellular networks as its future studies.

\section{Data Availability}

This study did not require any data.

\section{Conflicts of Interest}

The authors declare that there are no conflicts of interest in association with the publication of this article.

\section{References}

[1] O. Kainz, M. Michalko, F. Jakab, R. Petija, J. Uramova, and M. Moravcik, "Innovation in educational process of computer networks," in 2019 17th International Conference on Emerging eLearning Technologies and Applications (ICETA), pp. 336341, Starý Smokovec, Slovakia, 2019.
[2] Y. Wang, Z. Wang, X. Hu, T. Bai, S. Yang, and L. Huang, "A courses ontology system for computer science education," in 2019 IEEE International Conference on Computer Science and Educational Informatization (CSEI), pp. 251-254, Kunming, China, 2019.

[3] W.-X. Cai, G.-S. Li, X.-H. Chen et al., "Education based new computer network simulator design and implementation," in 2016 11th International Conference on Computer Science \& Education (ICCSE), pp. 933-935, Nagoya, Japan, 2016.

[4] E. A. Walelgne, A. S. Asrese, J. Manner, V. Bajpai, and J. Ott, "Understanding data usage patterns of geographically diverse mobile users," IEEE Transactions on Network and Service Management, vol. 18, no. 3, pp. 3798-3812, 2021.

[5] Y.-T. Lai, Y.-P. Wu, C.-H. Yu, F.-S. Lu, and C.-H. Chen, "Mobile data usage prediction system and method," in 2017 31st International Conference on Advanced Information Networking and Applications Workshops (WAINA), pp. 484-486, Taipei, Taiwan, 2017.

[6] GSMA, GSMA, The Mobile Economy 2019, 2019, Retrieved from GSMA: https://data.gsmaintelligence.com/api-web/v2/ research-file-download?id=39256194\&file $=2712-250219-\mathrm{ME}-$ Global.pdf.

[7] A. Habbal, S. I. Goudar, and S. Hassan, "A context-aware radio access technology selection mechanism in $5 \mathrm{G}$ mobile network for smart city applications," Journal of Network and Computer Applications, vol. 135, pp. 97-107, 2019.

[8] M. Lauridsen, L. C. Gimenez, I. Rodriguez, T. B. Sorensen, and P. Mogensen, "From LTE to 5G for connected mobility," IEEE Communications Magazine, vol. 55, no. 3, pp. 156-162, 2017.

[9] M. Ayad, S. Medjedoub, B. Mourad, K. Saoudi, and A. Arabi, "Evaluation of 4G/LTE mobile network performances based on experimental data," in 2020 2nd International Workshop 
on Human-Centric Smart Environments for Health and Wellbeing (IHSH), pp. 137-141, Boumerdes, Algeria, 2021.

[10] R. Zeqiri, F. Idrizi, and H. Halimi, "Comparison of algorithms and technologies 2G, 3G, 4G and 5G," in 2019 3rd International Symposium on Multidisciplinary Studies and Innovative Technologies (ISMSIT), Ankara, Turkey, 2019.

[11] P. Neog and R. Bera, "Multi-standard radio for $2 \mathrm{G}$ to $5 \mathrm{G}$," in 2017 2nd International Conference on Telecommunication and Networks (TEL-NET), Noida, India, 2017.

[12] E. Dahlman, S. Parkvall, and J. Sköld, 4G, LTE-advanced pro and the road to 5G, Academic Press, Third edition, 2016.

[13] S. J. Nawaz, S. K. Sharma, S. Wyne, M. N. Patwary, and M. Asaduzzaman, "Quantum machine learning for 6G communication networks: state-of-the-art and vision for the future," IEEE Access, vol. 7, pp. 46317-46350, 2019.

[14] K. Riyazuddin, D. A. Sharma, and D. P. Reddy, "Analyzing the behaviour of OFDM parameters in different LTE environment," in 2017 IEEE International Conference on Power, Control, Signals and Instrumentation Engineering (ICPCSI), pp. 2849-2853, Chennai, India, 2017.

[15] J. Li, M. Wen, X. Jiang, and Y. Yan, "Novel control signal detection for high-rate transmission in LTE-OFDM systems," in 2016 IEEE International Conference on Signal Processing, Communications and Computing (ICSPCC), Hong Kong, China, 2016.

[16] B. Xia, Y. Wang, and G. Yang, "Data-aided carrier frequency offset estimation for orthogonal frequency division multiplexing communication systems," in International Conference on Computer and Communications, pp. 848-852, Chengdu, China, 2019.

[17] M. B. Rossberg, G. Schaefer, and D. Sukiennik, "A comprehensive framework to evaluate wireless networks in simulation and real systems," in 2018 IEEE/ACM 22nd International Symposium on Distributed Simulation and Real Time Applications (DS-RT), Madrid, Spain, 2018.

[18] R. F. Sari and R. Harwahyu, "Teaching internet protocol engineering with open source simulators: a long road from WAN to 5G," in 2019 IEEE 11th International Conference on Engineering Education (ICEED), pp. 122-127, Kanazawa, Japan, 2019.

[19] M. Bazdresch, "A small network simulator for learning routing fundamentals," in 2018 IEEE Frontiers in Education Conference (FIE), San Jose, CA, USA, 2018.

[20] M. Pezer, N. Lazić, and M. Odak, "Free and open source software in the secondary education in Bosnia and Herzegovina," in 2017 40th International Convention on Information and Communication Technology, Electronics and Microelectronics (MIPRO), pp. 882-886, Opatija, Croatia, 2017.

[21] D. A. Almeida, G. C. Murphy, G. Wilson, and M. Hoye, "Do software developers understand open source licenses?," in 2017 IEEE/ACM 25th International Conference on Program Comprehension (ICPC), Buenos Aires, Argentina, 2017.

[22] D. C. Wynn and P. J. Clarkson, "Process models in design and development," Research in Engineering Design, vol. 29, no. 2, pp. 161-202, 2018.

[23] Openxcell, What is SDLC (Software Development Life Cycle)?, 2020, Retrieved from https://www.openxcell.com/blog/ software-development-life-cycle/. 